\title{
O RESFRIAMENTO RÁPIDO E A RÁPIDA INSTALAÇÃO DA ATMOSFERA CONTROLADA COMO SUBSTITUTOS DO 1-MCP NO ARMAZENAMENTO DE MAÇÃS 'GALA'
}

\author{
AURI BRACKMANN², RICARDO FABIANO HETTWER GIEHL ${ }^{3}$, SÉRGIO TONETTO DE FREITAS², \\ ANDERSON MACHADO DE MELLO ${ }^{4}$, ROSE BEATRIZ ANTES ${ }^{5}$
}

\begin{abstract}
RESUMO - O objetivo deste trabalho foi avaliar o efeito da aplicação do 1-metilciclopropeno (1-MCP) associada a diferentes formas de resfriamento e de instalação da atmosfera controlada (AC) sobre a qualidade da maçã 'Gala'. No primeiro experimento, os tratamentos consistiram da aplicação ou não de 1-MCP no início do armazenamento refrigerado com dois períodos necessários para o resfriamento dos frutos até a temperatura de armazenamento $\left(0,5^{\circ} \mathrm{C}\right)$ : sete dias (lento) e três dias (rápido). No segundo experimento, avaliou-se o efeito da aplicação de 1-MCP associada com a

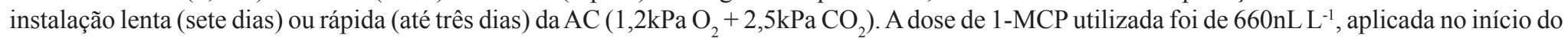
armazenamento, durante 24 horas, a $0,5^{\circ} \mathrm{C}$. No primeiro ensaio, o resfriamento dos frutos em três dias, em comparação com o resfriamento em sete dias, permitiu melhor manutenção dos atributos qualitativos dos frutos após seis meses de armazenamento refrigerado a $0,5^{\circ} \mathrm{C}$ e mais sete dias a $20^{\circ} \mathrm{C}$, independentemente da aplicação de 1-MCP. No segundo experimento, observou-se que a instalação rápida da AC proporcionou resultados semelhantes à aplicação de 1-MCP, durante a instalação lenta, na manutenção da qualidade dos frutos, após oito meses de armazenamento em AC, a $0,5^{\circ} \mathrm{C}$, e mais sete dias a $20^{\circ} \mathrm{C}$. Esses resultados sugerem que o resfriamento rápido ou a rápida instalação da $\mathrm{AC}$ podem substituir a aplicação de 1-MCP em maçãs 'Gala'.
\end{abstract}

Termos para indexação: Malus domestica Borkh., 1-MCP, armazenamento, etileno.

\section{FAST COOLING AND RAPID CONTROLLED ATMOSPHERE AS SUBSTITUTE OF 1-MCP ON 'GALA' APPLE FRUITS STORAGE}

\begin{abstract}
The aim of this work was to evaluate the effect of 1-methylcyclopropene (1-MCP) application associated with different rates of cooling and settlement of controlled stored atmosphere (CA) on quality of 'Gala' apple fruits. In the first experiment, the treatments were with or without 1-MCP and two times to reach the storage temperature $\left(0.5^{\circ} \mathrm{C}\right): 7$ days (slow) and 3 days (fast). The second experiment evaluated the effect of 1-MCP application and the settlement of stored CA: slow ( 7 days) and fast ( 3 days). The CA condition was $1.2 \mathrm{kPa} \mathrm{O}_{2}+2.5 \mathrm{kPa} \mathrm{CO}{ }_{2}$ at $0.5^{\circ} \mathrm{C}$. It was applied $660 \mathrm{~nL} \mathrm{~L}^{-1}$ of $1-\mathrm{MCP}$ during $24 \mathrm{~h}$ at $0.5^{\circ} \mathrm{C}$ in the beginning of storage on both experiments. In the first experiment fast-cooled fruit had better quality than slow-cooled fruit, independently of 1-MCP application following 6 months of storage at $0.5^{\circ} \mathrm{C}$ plus 7 days at $20^{\circ} \mathrm{C}$. Results obtained in the second experiment showed that after 8 months of CA storage at $0.5^{\circ} \mathrm{C}$ plus 7 days of shelf-life at $20^{\circ} \mathrm{C}$, the fast CA allowed a similar quality to 1 $\mathrm{MCP}$-treatment plus slow CA-stored fruit. These results suggest that fast cooling or fast CA can substitute 1-MCP application on 'Gala' apple fruits. Index terms: Malus domestica Borkh., 1-MCP, storage, ethylene.
\end{abstract}

\section{INTRODUÇÃO}

A maçã 'Gala', em função de sua elevada taxa respiratória e alta produção de etileno, apresenta rápida perda de firmeza da polpa, redução da acidez titulável e amarelecimento da cor de fundo da epiderme, durante o armazenamento e o processo de comercialização.

O rápido resfriamento dos frutos é uma prática recomendada, por reduzir rapidamente a atividade metabólica. Segundo Lau \& Looney (1982), o atraso no processo de resfriamento ocasiona perdas nos atributos qualitativos dos frutos. Em maçãs 'McIntosh', o resfriamento dos frutos em um dia reduz a síntese de etileno e a perda de firmeza, em comparação com o resfriamento em quatro dias (Fica et al., 1985).

$\mathrm{O}$ armazenamento em atmosfera controlada (AC) prolonga o período de armazenamento. Práticas associadas ao uso de AC, como o período necessário para a instalação das pressões parciais de $\mathrm{O}_{2} \mathrm{e} \mathrm{CO}_{2}$, vêm sendo investigadas como formas de incrementar ainda mais o efeito do controle dos gases. De acordo com Brackmann et al. (2000), a rápida instalação da AC reduz a perda da firmeza da polpa, o amarelecimento da epiderme e a ocorrência de polpa farinhenta durante o armazenamento de maçãs 'Gala'.

O 1-metilciclopropeno (1-MCP ou $\mathrm{C}_{4} \mathrm{H}_{6}$ ) é um composto volátil, que tem demonstrado ser um potente inibidor da ação do etileno (Serek et al., 1995), fitormônio responsável pelo amadurecimento dos frutos. De acordo com Blankenship \& Dole (2003), o 1-MCP liga-se aos receptores de etileno, impedindo a ligação e a ação dos mesmos. Como resultado, o estímulo fisiológico e as rotas de transdução de sinal são inibidos, influenciando no processo de amadurecimento dos frutos. Trabalhos realizados com a aplicação de 1-MCP demonstraram que esse composto diminuiu a síntese de etileno, a respiração e manteve maiores valores de firmeza da polpa, acidez titulável e sólidos solúveis totais em maçãs 'Fuji', 'Granny Smith' e 'Gala' (Fan et al., 1999a), além de reduzir a incidência de podridões (Mir \& Beaudry, 2000) e de distúrbios fisiológicos, como a escaldadura e o escurecimento interno em maçãs (Fan et al., 1999b).

Apesar do grande número de trabalhos conduzidos até o momento avaliando o efeito do 1-MCP, não há informações comparativas entre esse tratamento e a instalação rápida da $\mathrm{AC}$ e o resfriamento rápido dos frutos. Portanto, o objetivo deste trabalho foi avaliar o efeito da aplicação do 1-MCP com diferentes formas de resfriamento e de instalação da atmosfera controlada (AC) sobre a qualidade da maçã 'Gala'.

\section{MATERIAL E MÉTODOS}

Este trabalho constituiu-se de dois experimentos, que foram conduzidos no Núcleo de Pesquisa em Pós-Colheita do Departamento de Fitotecnia da Universidade Federal de Santa Maria. O delineamento experimental utilizado, para ambos os experimentos, foi o inteiramente casualizado, sendo os tratamentos constituídos por cinco repetições de 25 frutos.

\footnotetext{
(Trabalho 027/2005). Recebido: 23/02/2005. Aceito para publicação: 17/11/2005.

${ }^{2}$ Engenheiro Agrônomo, Doutor em Ciências Agrárias, Professor Adjunto do Departamento de Fitotecnia da Universidade Federal de Santa Maria (UFSM), 97.105-

900, Santa Maria-RS. E-mail: brackman@ccr.ufsm.br (autor para correspondência).

${ }^{3}$ Engenheiro Agrônomo, aluno do PPGA da UFSM. Bolsista CNPq.

${ }^{4}$ Engenheiro Agrônomo, M. Sc. aluno do PPGA da UFSM. Bolsista CNPq.

${ }^{5}$ Engenheira Agrônoma. Departamento de Fitotecnia da UFSM.
} 
No experimento 1 , os tratamentos originaram-se da combinação da aplicação ou não de $660 \mathrm{~nL} \mathrm{~L}^{-1}$ de $1-\mathrm{MCP}$, no início do armazenamento refrigerado, com dois períodos necessários para resfriamento dos frutos até a temperatura de armazenamento $\left(0,5^{\circ} \mathrm{C}\right)$ : sete dias (lento) e três dias (rápido). A temperatura inicial na polpa dos frutos foi de $24,5 \pm 1^{\circ} \mathrm{C}$. O resfriamento lento foi efetuado em uma câmara frigorífica, por meio de ventilação forçada e constante. Para que a polpa dos frutos atingisse a temperatura de armazenamento em sete dias, baixou-se diariamente a temperatura da câmara em $3,5^{\circ} \mathrm{C}$. Já no resfriamento rápido, baixou-se a temperatura da câmara em $8^{\circ} \mathrm{C} \mathrm{a}$ cada dia, para que a temperatura da polpa dos frutos alcançasse $0,5^{\circ} \mathrm{C}$ em três dias. $\mathrm{O}$ resfriamento lento simulou o método adotado pelas empresas de armazenamento de maçãs, que costumam prolongar o resfriamento dos frutos por mais de seis dias. O monitoramento da temperatura dos frutos durante o resfriamento foi efetuado com o auxílio de termômetros de bulbo de mercúrio da marca Incoterm $^{\circledR}$, com precisão de $0,1^{\circ} \mathrm{C}$, inseridos na polpa de frutos até próximo ao carpelo. Esses frutos foram armazenados, durante seis meses, em minicâmaras experimentais de $0,233 \mathrm{~m}^{3}$, sob condições de ambiente refrigerado a $0,5 \pm 0,2^{\circ} \mathrm{C}$ com $95 \pm 1 \%$ de umidade relativa do ar.

No experimento 2, os tratamentos avaliados foram compostos pela combinação da aplicação ou não de $660 \mathrm{~nL} \mathrm{~L}^{-1}$ de $1-\mathrm{MCP}$, no início do armazenamento, e duas velocidades de instalação da $\mathrm{AC}$ (rápida e lenta). $\mathrm{Na}$ instalação lenta da $\mathrm{AC}$, foram necessários sete dias para atingir as pressões parciais de $\mathrm{O}_{2}$ e $\mathrm{CO}_{2}$ preestabelecidas. Já na instalação rápida, esse período foi reduzido para apenas três dias. A instalação lenta da $\mathrm{AC}$ simulou o procedimento adotado nas empresas brasileiras que, devido ao grande volume de frutos armazenados e custo do $\mathrm{N}_{2}$ e do $\mathrm{CO}_{2}$, não conseguem instalar de forma rápida as condições de armazenamento.

Os frutos do segundo experimento foram armazenados em

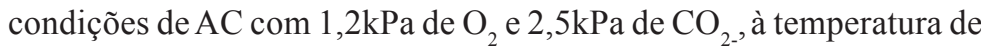
$0,5^{\circ} \mathrm{C}$. Os níveis de $\mathrm{O}_{2}$, para ambas as formas de instalação da $\mathrm{AC}$, foram obtidos através da injeção, nas minicâmaras, de $\mathrm{N}_{2}$ oriundo de um equipamento gerador de $\mathrm{N}_{2}$, que funciona pelo princípio "Pressure Swing Adsorption" (PSA). Na instalação rápida da $\mathrm{AC}$, as pressões parciais de $\mathrm{CO}_{2}$ foram obtidas mediante a injeção deste gás nas minicâmaras, de modo que as pressões parciais desejadas fossem atingidas em três dias. $\mathrm{Na}$ instalação lenta, a pressão parcial de $\mathrm{CO}_{2}$ foi obtida pelo processo respiratório dos frutos, sendo que, após sete dias, as pressões parciais de $\mathrm{O}_{2}$ e $\mathrm{CO}_{2}$ preestabelecidas foram alcançadas.

As pressões parciais de $\mathrm{O}_{2}$ e $\mathrm{CO}_{2}$, nas minicâmaras experimentais, foram analisadas e corrigidas diversas vezes ao dia por um equipamento de controle automático de gases (marca Kronenberger). As temperaturas das câmaras frigoríficas foram reguladas automaticamente, através de termostatos eletrônicos, sendo acompanhadas diariamente por meio de termômetros de bulbo de mercúrio da marca Incoterm ${ }^{\circledR}$, com precisão de $0,1^{\circ} \mathrm{C}$, inseridos na polpa de frutos até próximo ao carpelo.

Para ambos os experimentos, o 1-MCP, na dose de $660 \mathrm{~nL} \mathrm{~L}^{-1}$, foi aplicado nos frutos em minicâmaras $\left(0,233 \mathrm{~m}^{3}\right)$, hermeticamente fechadas, durante 24 horas, sob $0,5^{\circ} \mathrm{C}$. Utilizou-se como fonte do 1 MCP o produto $\operatorname{SmartFresh}^{\circledR}(0,43 \%$ de 1 -MCP na formulação pó).

As análises laboratoriais foram realizadas após seis e oito meses de armazenamento, para o primeiro e segundo experimentos, respectivamente, sendo realizadas no momento da saída dos frutos das câmaras e após sete dias de exposição à temperatura de $20^{\circ} \mathrm{C}$. As variáveis avaliadas foram: firmeza da polpa, acidez titulável, sólidos solúveis totais (SST), cor de fundo da epiderme, taxa de produção de $\mathrm{CO}_{2-}$ e produção de etileno, conforme descrito em Brackmann et al. (2004). Os resultados foram submetidos à análise da variância, sendo as médias comparadas entre si, pelo teste de Duncan, em nível de 5\% de probabilidade de erro.

\section{RESULTADOS E DISCUSSÃO}

Em relação ao primeiro experimento, verificou-se que, após seis meses de armazenamento refrigerado a $0,5^{\circ} \mathrm{C}$, o resfriamento dos frutos, em três dias, associado à aplicação de 1-MCP, permitiu maiores valores de firmeza da polpa e de acidez titulável na saída dos frutos das câmaras e após sete dias a $20^{\circ} \mathrm{C}$ (Tabela 1). No entanto, esses resultados foram estatisticamente semelhantes ao resfriamento em três dias sem aplicação de 1-MCP, evidenciando um maior efeito do resfriamento rápido dos frutos, na manutenção da firmeza da polpa e da acidez titulável, em comparação à aplicação de 1-MCP. Brackmann et al. (2000) verificaram que o abaixamento imediato da temperatura promove maior retenção da firmeza da polpa de maçãs. O 1-MCP também apresentou efeito positivo sobre a manutenção da firmeza da polpa e da acidez titulável de maçãs 'Gala' (Fan et al., 1999a; Brackmann et al., 2004).

Os valores de sólidos solúveis totais (SST) não diferiram estatisticamente na análise realizada na saída dos frutos da câmara (Tabela 1). No entanto, após sete dias a $20^{\circ} \mathrm{C}$, os frutos submetidos ao resfriamento em sete dias com 1-MCP e ao resfriamento em três dias com e sem 1-MCP apresentaram valores de SST mais elevados, quando comparados àqueles resfriados lentamente sem 1-MCP (Tabela 1).

Em relação à cor de fundo da epiderme, houve diferença

TABELA 1 - Qualidade física e química de maçãs 'Gala' submetidas à aplicação de 1-MCP associada a diferentes formas de resfriamento, após seis meses de armazenamento refrigerado a $0,5^{\circ} \mathrm{C}$ e mais sete dias a $20^{\circ} \mathrm{C}$. Santa Maria-RS.

\begin{tabular}{|c|c|c|c|c|c|c|c|}
\hline Resfriamento* & $\begin{array}{c}\text { 1-MCP } \\
\left(660 \mathrm{~nL} \mathrm{~L}^{-1}\right)\end{array}$ & $\begin{array}{c}\text { Firmeza de } \\
\text { polpa } \\
(\mathrm{N}) \\
\end{array}$ & $\begin{array}{l}\text { Acidez titulável } \\
\left(\text { meq } 100 \mathrm{~mL}^{-1}\right)\end{array}$ & $\begin{array}{c}\text { SST } \\
\left({ }^{\circ} \text { Brix }\right)\end{array}$ & $\begin{array}{l}\text { Cor fundo } \\
\text { epiderme } \\
\left(a^{*}+b^{*}\right)\end{array}$ & $\begin{array}{c}\text { Respiração } \\
\left(\mathrm{mlCO}_{2} \mathrm{~kg} \mathrm{~h}^{-1}\right)\end{array}$ & $\begin{array}{c}\text { Produção de } \\
\text { etileno } \\
\left(\mu \mathrm{Lg} \mathrm{h}^{-1}\right) \\
\end{array}$ \\
\hline & & \multicolumn{6}{|c|}{ Saída da câmara } \\
\hline Lento & Sem & $50,2 \quad \mathrm{c}^{* *}$ & $2,92 \mathrm{~b}$ & $11,9 \mathrm{a}$ & $56,8 \mathrm{a}$ & $19,50 \mathrm{a}$ & $202,70 \mathrm{a}$ \\
\hline Lento & Com & 52,9 bc & $2,97 \mathrm{~b}$ & $11,7 \mathrm{a}$ & $56,2 \mathrm{a}$ & $12,80 \mathrm{~b}$ & $135,90 \mathrm{~b}$ \\
\hline Rápido & Sem & $59,2 \mathrm{abc}$ & $3,18 \mathrm{ab}$ & $11,8 \mathrm{a}$ & $55,1 \mathrm{a}$ & $12,60 \mathrm{~b}$ & $150,00 \mathrm{~b}$ \\
\hline Rápido & Com & $65,4 \mathrm{a}$ & $3,53 \mathrm{a}$ & $12,7 \mathrm{a}$ & 54,6 a & $7,73 \mathrm{c}$ & $1,79 \mathrm{c}$ \\
\hline \multicolumn{2}{|c|}{ C.V. $(\%)$} & 2,89 & 9,85 & 1,73 & 2,69 & 8,62 & 8,49 \\
\hline & & \multicolumn{6}{|c|}{ Após sete dias a $20^{\circ} \mathrm{C}$} \\
\hline Lento & Sem & $44,9 \mathrm{~b}$ & $2,45 \mathrm{~b}$ & $11,7 \mathrm{~b}$ & $60,6 \mathrm{a}$ & $12,9 \mathrm{a}$ & $97,50 \mathrm{a}$ \\
\hline Lento & Com & $49,6 \mathrm{~b}$ & $2,67 \mathrm{ab}$ & $12,4 \mathrm{a}$ & $61,4 \mathrm{a}$ & $7,85 \mathrm{~b}$ & $85,80 \mathrm{ab}$ \\
\hline Rápido & Sem & $57,8 \mathrm{a}$ & $2,73 \mathrm{a}$ & $12,4 \mathrm{a}$ & $61,7 \mathrm{a}$ & $6,20 \mathrm{~b}$ & $73,40 \quad b$ \\
\hline Rápido & Com & $62,9 \mathrm{a}$ & $2,75 \mathrm{a}$ & $12,1 \mathrm{ab}$ & $55,9 \mathrm{~b}$ & $4,07 \quad \mathrm{c}$ & $9,95 \quad \mathrm{c}$ \\
\hline \multicolumn{2}{|c|}{ C.V. $(\%)$} & 6,41 & 5,97 & 2,86 & 4,46 & 15,80 & 13,60 \\
\hline
\end{tabular}

* Resfriamento lento: sete dias para a polpa dos frutos atingirem a temperatura de armazenamento; Resfriamento rápido: três dias para a polpa dos frutos atingirem a temperatura de armazenamento.

** Médias seguidas pela mesma letra não diferem entre si, pelo teste de Duncan, em nível de 5\% de probabilidade de erro. 
TABELA 2 - Qualidades físico-químicas de maçãs 'Gala' submetidas à aplicação de 1-MCP associada a diferentes formas de instalação da AC (1,2kPa $\mathrm{O}_{2}+2,5 \mathrm{kPa} \mathrm{CO}_{2}$ ), após seis meses de armazenamento a $0,5^{\circ} \mathrm{C}$ e mais sete dias a $20^{\circ} \mathrm{C}$. Santa Maria-RS.

\begin{tabular}{|c|c|c|c|c|c|c|c|}
\hline $\begin{array}{c}\text { Instalação da } \\
\text { atmosfera } \\
\text { controlada* }\end{array}$ & $\begin{array}{c}\text { 1-MCP } \\
\left(660 \mathrm{~nL} \mathrm{~L}^{-1}\right)\end{array}$ & $\begin{array}{c}\text { Firmeza de } \\
\text { polpa } \\
(\mathrm{N})\end{array}$ & $\begin{array}{l}\text { Acidez titulável } \\
\left(\text { meq } 100 \mathrm{~mL}^{-1}\right)\end{array}$ & $\begin{array}{c}\text { SST } \\
\left({ }^{\circ} \text { Brix }\right)\end{array}$ & $\begin{array}{l}\text { Cor fundo } \\
\text { epiderme } \\
\left(a^{*}+b^{*}\right)\end{array}$ & $\begin{array}{c}\text { Respiração } \\
\left(\mathrm{ml} \mathrm{CO}_{2} \mathrm{~kg} \mathrm{~h}^{-1}\right)\end{array}$ & $\begin{array}{c}\text { Produção de } \\
\text { etileno } \\
\left(\mu \mathrm{Lkg} \mathrm{h}^{-1}\right)\end{array}$ \\
\hline & & \multicolumn{6}{|c|}{ Saída da câmara } \\
\hline Lenta & Sem & $59,4 \quad b^{* *}$ & $4,56 \mathrm{~b}$ & $12,6 \mathrm{a}$ & $50,0 \mathrm{a}$ & $11,70 \mathrm{a}$ & $19,3 \mathrm{a}$ \\
\hline Lenta & Com & $67,1 \mathrm{a}$ & $4,96 \mathrm{ab}$ & $12,2 \mathrm{a}$ & $44,9 \quad b$ & $10,00 \mathrm{ab}$ & $0,66 \mathrm{~b}$ \\
\hline Rápida & Sem & $62,7 \mathrm{ab}$ & $5,52 \mathrm{a}$ & $12,1 \mathrm{a}$ & $46,2 \mathrm{ab}$ & $8,40 \mathrm{~b}$ & $18,4 \mathrm{a}$ \\
\hline Rápida & Com & $67,0 \mathrm{a}$ & $5,33 \mathrm{ab}$ & $12,2 \mathrm{a}$ & $46,7 \mathrm{ab}$ & $8,88 \mathrm{~b}$ & $0,89 \mathrm{~b}$ \\
\hline C.V. $(\%)$ & & 6,77 & 9,86 & 3,55 & 6,12 & 16,77 & 33,52 \\
\hline \multicolumn{8}{|c|}{ Após sete dias a $20^{\circ} \mathrm{C}$} \\
\hline Lenta & Sem & 53,3 a & $4,52 \mathrm{a}$ & $12,5 \mathrm{a}$ & $51,5 \mathrm{ab}$ & $41,2 \mathrm{a}$ & $2,07 \mathrm{a}$ \\
\hline Lenta & Com & $57,2 \mathrm{a}$ & $4,44 \mathrm{a}$ & $12,4 \mathrm{a}$ & 49,8 bc & $14,0 \mathrm{~b}$ & $1,28 \mathrm{a}$ \\
\hline Rápida & Sem & $57,8 \mathrm{a}$ & $4,27 \mathrm{a}$ & $12,4 \mathrm{a}$ & $54,6 \mathrm{a}$ & $7,16 \mathrm{~b}$ & $2,18 \mathrm{a}$ \\
\hline Rápida & Com & 63,3 a & $4,37 \mathrm{a}$ & $12,3 \mathrm{a}$ & $46,4 \quad \mathrm{c}$ & $8,38 \mathrm{~b}$ & $0,27 \mathrm{a}$ \\
\hline C.V. $(\%)$ & & 11,80 & 4,96 & 6,72 & 4,72 & 48,61 & 43,40 \\
\hline
\end{tabular}

* Pressões parciais de $1,2 \mathrm{kPa} \mathrm{O}_{2}$ e $2,5 \mathrm{kPa} \mathrm{CO}_{2}$, instaladas em sete dias (instalação lenta) e em três dias (instalação rápida), após o fechamento das câmaras;

** Médias seguidas pela mesma letra não diferem entre si, pelo teste de Duncan, em nível de $5 \%$ de probabilidade de erro.

estatística significativa somente após sete dias a $20^{\circ} \mathrm{C}$, quando os frutos resfriados em três dias e tratados com de 1-MCP apresentaram uma coloração da epiderme mais verde em relação aos demais (Tabela 1). Possivelmente, esse resultado esteja relacionado à redução do metabolismo no início do armazenamento, pelo menor período necessário para o resfriamento dos frutos, associado com a inibição da ação do etileno pelo 1-MCP, pois a exposição dos frutos ao etileno acelera a degradação das clorofilas (Kader, 1985).

A produção de $\mathrm{CO}_{2-}$ e de etileno foi menor nos frutos tratados com 1-MCP e resfriados em três dias, na saída da câmara e após a exposição a $20^{\circ} \mathrm{C}$ (Tabela 1). Além disso, observou-se que a aplicação de 1-MCP, em frutos resfriados em sete dias, teve efeito igual estatisticamente ao resfriamento em três dias, sem a aplicação de 1$\mathrm{MCP}$, na redução da produção de $\mathrm{CO}_{2}$ e na síntese de etileno (Tabela 1). A aplicação de 1-MCP em maçãs armazenadas em baixas temperaturas promove redução na respiração e na produção de etileno, além de proporcionar melhor retenção da firmeza da polpa e da acidez titulável, conseqüentes da redução do metabolismo dos frutos (Fan et al., 1999a,b). Fica et al. (1985) observaram menor produção de etileno em maçãs 'McIntosh' resfriadas em um dia, em comparação com o resfriamento em quatro dias.

Em relação ao segundo experimento, verificou-se que a instalação rápida da $\mathrm{AC}$, com e sem a aplicação de 1-MCP e a instalação lenta com 1-MCP proporcionaram frutos com firmeza da polpa e acidez titulável mais elevadas na saída da câmara (Tabela 2). Esses resultados sugerem que a instalação rápida da $\mathrm{AC}$ permite um efeito semelhante à aplicação de $1-\mathrm{MCP}$. Após sete dias a $20^{\circ} \mathrm{C}$, não se verificou diferença estatística entre os tratamentos, em relação à firmeza da polpa e à acidez titulável (Tabela 2). Fan et al. (1999a) observaram acidez titulável mais elevada em maçãs tratadas com 1-MCP, em relação às não tratadas.

Os frutos tratados com 1-MCP apresentaram cor de fundo da epiderme mais verde que os não-tratados, após sete dias de exposição a $20^{\circ} \mathrm{C}$ (Tabela 2). Redução no amarelecimento da epiderme de maçãs 'Fuji' e 'Gala' foi observada com a aplicação de 1-MCP por Fan \& Mattheis (1999) e Brackmann et al. (2004), respectivamente.

Uma menor produção de $\mathrm{CO}_{2}$ pelos frutos, na saída da câmara, foi obtida com a instalação rápida da $\mathrm{AC}$ (Tabela 2). Após a exposição a $20^{\circ} \mathrm{C}$, os frutos submetidos à instalação lenta da $\mathrm{AC}$ e tratados com 1-MCP e os submetidos à instalação rápida da $\mathrm{AC}$, com ou sem 1MCP, apresentaram menor respiração (Tabela 2). A instalação rápida da $\mathrm{AC}$ isoladamente proporcionou resultados iguais à aplicação de 1$\mathrm{MCP}$, associada à instalação lenta da atmosfera. Redução na respiração de maçãs foram reportadas por Fan et al. (1999a), com a aplicação de 1-MCP.

A aplicação de 1-MCP reduziu a produção de etileno, na saída dos frutos da câmara, independentemente do período necessário para a instalação da AC (Tabela 2). Esses resultados concordam com os reportados por Fan \& Mattheis (1999) e Fan et al. (1999a,b). Após a exposição dos frutos a $20^{\circ} \mathrm{C}$, não se observou diferença significativa entre os tratamentos avaliados (Tabela 2).

\section{CONCLUSÕES}

1. O resfriamento dos frutos em três, em comparação com o resfriamento em sete dias, permite melhor manutenção dos atributos qualitativos de maçãs 'Gala', independentemente da aplicação de $660 \mathrm{~nL} \mathrm{~L}^{-1}$ de 1-MCP, após um período de seis meses de armazenamento a $0,5^{\circ} \mathrm{C}$ e mais sete dias a $20^{\circ} \mathrm{C}$.

2. A instalação das condições de atmosfera controlada $(1,2 \mathrm{kPa}$ $\mathrm{O}_{2}+2,5 \mathrm{kPa} \mathrm{CO} \mathrm{CO}_{2}$ ) em até três dias (instalação rápida) proporciona resultados semelhantes à aplicação de $660 \mathrm{~nL} \mathrm{~L}^{-1}$ de $1-\mathrm{MCP}$ com a instalação da $\mathrm{AC}$ em sete dias (instalação lenta), em relação à manutenção da qualidade de maçãs 'Gala', após oito meses de armazenamento em $\mathrm{AC} \mathrm{a} 0,5^{\circ} \mathrm{C}$ e mais sete dias a $20^{\circ} \mathrm{C}$.

\section{REFERÊNCIAS}

BLANKENSHIP, S.M.; DOLE, J.M. 1-Methylcyclopropene: a review. Postharvest Biology and Technology, Amsterdam, v.28, p.1-25, 2003.

BRACKMANN, A.; HUNSCHE, M.; STEFFENS, C.A. Qualidade da maçã 'Gala' (Malus domestica Borkh.) submetida a diferentes períodos de resfriamento dos frutos e concentração de etileno. Revista Brasileira de Fruticultura, Jaboticabal, v.22, n. especial, p. 01-05, 2000.

BRACKMANN, A.; SESTARI, I.; STEFFENS, C.A.; GIEHL, R.F.H. Qualidade da maçã cv. Gala tratada com 1-metilciclopropeno. Ciência Rural, Santa Maria, v.34, n.5, p.1.415-1.420, 2004.

FAN, X.; BLANKENSHIP, S.M.; MATTHEIS, J.P. 1Methylcyclopropene inhibits apple ripening. Journal of the American Society for Horticultural Science, Alexandria, v.124, n.6, p.690-695, 1999a.

FAN, X.; MATTHEIS, J.P. Methyl jasmonate promotes apple fruit degreening independently of ethylene action. HortScience, Alexandria, v.34, p.310-312, 1999.

FAN, X.; MATTHEIS, J.P.; BLANKENSHIP, S.M. Development of apple superficial scald, soft scald, core flush, and greasiness is reduced by MCP. Journal of Agricultural and Food Chemistry, Washington, v.47, n.8, p.3.063-3.068, 1999b. 
FICA, J.; SKRZYNSKI, J.; DILLEY, D.R. The effect of delayed cooling and delayed application of CA storage of McIntosh apples under low and high ethylene levels. In: NATIONAL CONTROLLED ATMOSPHERE RESEARCH CONFERENCE, 4., 1985, Raleigh. Proceedings... v.4, p.82-94.

KADER, A.A. Ethylene-induced senescence and physiological disorders in harvested horticultural crops. HortScience, Alexandria, v.20, n.1, p.54-56, 1985.

LAU, O.L.; LOONEY, N.E. Improvement of fruit firmness and acidity in controlled-atmosphere-stored 'Golden Delicious' apples by a rapid $\mathrm{O}_{2}$ reduction procedure. Journal of American Society Horticultural Science, Alexandria, n.107, p.531-534, 1982.
MIR, N.A.; BEAUDRY, R.M. Use of 1-MCP to reduce the requirement for refrigeration and modified-atmospheres in the storage of apple fruit. In.: INTERNATIONAL CONFERENCE ON POSTHARVEST SCIENCE, 2000, Jerusalem. Abstracts..., Jerusalem, Israel: ISHS/ ARO, 2000.p.23

SEREK, M.; SISLER, E.C.; REID, M.S. 1-metylcyclopropene, a novel gaseous inhibitor of ethylene action, improves the life of fruit, cut flowers and potted plants. Acta Horticulturae, Leuven, n.394, p.337-345, 1995. 